\title{
Development of an airway mucus defect in the cystic fibrosis rat
}

\author{
Susan E. Birket, ${ }^{1,2}$ Joy M. Davis, ${ }^{1}$ Courtney M. Fernandez, ${ }^{1}$ Katherine L. Tuggle, ${ }^{2}$ Ashley M. Oden, ${ }^{1}$ \\ Kengyeh K. Chu, ${ }^{3}$ Guillermo J. Tearney, ${ }^{3,4,5}$ Michelle V. Fanucchi, ${ }^{6}$ Eric J. Sorscher, ${ }^{7}$ \\ and Steven M. Rowe $e^{1,2,8,9}$ \\ 'Department of Medicine and 2Cystic Fibrosis Research Center, University of Alabama at Birmingham, Birmingham, \\ Alabama, USA. ${ }^{3}$ Wellman Center for Photomedicine, Massachusetts General Hospital, Boston, Massachusetts, USA. \\ ${ }^{4}$ Harvard Medical School, Boston, Massachusetts, USA. ${ }^{5}$ Harvard-MIT Division of Health Sciences and Technology, \\ Cambridge, Massachusetts, USA. ${ }^{6}$ Department of Environmental Health Sciences, University of Alabama at Birmingham, \\ Birmingham, Alabama, USA. ${ }^{7}$ Department of Pediatrics, Emory University, Atlanta, Georgia, USA. ${ }^{8}$ Department of \\ Cellular, Developmental, and Integrative Biology and ${ }^{9}$ Department of Pediatrics, University of Alabama at Birmingham, \\ Birmingham, Alabama, USA.
}

The mechanisms underlying the development and natural progression of the airway mucus defect in cystic fibrosis (CF) remain largely unclear. New animal models of CF, coupled with imaging using micro-optical coherence tomography, can lead to insights regarding these questions. The $\mathrm{Cftr}^{-1}$ (KO) rat allows for longitudinal examination of the development and progression of airway mucus abnormalities. The $\mathrm{KO}$ rat exhibits decreased periciliary depth, hyperacidic $\mathrm{pH}$, and increased mucus solid content percentage; however, the transport rates and viscoelastic properties of the mucus are unaffected until the KO rat ages. Airway submucosal gland hypertrophy develops in the KO rat by 6 months of age. Only then does it induce increased mucus viscosity, collapse of the periciliary layer, and delayed mucociliary transport; stimulation of gland secretion potentiates this evolution. These findings could be reversed by bicarbonate repletion but not $\mathrm{pH}$ correction without counterion donation. These studies demonstrate that abnormal surface epithelium in CF does not cause delayed mucus transport in the absence of functional gland secretions. Furthermore, abnormal bicarbonate transport represents a specific target for restoring mucus clearance, independent of effects on periciliary collapse. Thus, mature airway secretions are required to manifest the CF defect primed by airway dehydration and bicarbonate deficiency.

Conflict of interest: K.K. Chu, G.J. Tearney, and S.M. Rowe hold a patent $(14 / 240,938)$ on the use of $\mu 0 \mathrm{OCT}$ for pharmaceutical drug discovery.

Submitted: August 31, 2017 Accepted: November 30, 2017 Published: January 11, 2018

\section{Reference information:} JCI Insight. 2018;3(1):e97199. https:// doi.org/10.1172/jii.insight.97199

\section{Introduction}

Cystic fibrosis (CF) is the most common autosomal recessive genetic disorder, resulting from dysfunction of the CF transmembrane conductance regulator (CFTR) protein (1). More than 1,700 disease-causing mutations in the CFTR have been identified, many of which result in little or no CFTR activity (2). CFTR transports both chloride and bicarbonate ions across the epithelial surface of many tissues (3-5); in the airway, this provides the epithelial surface with adequate hydration, osmotic pressure, and $\mathrm{pH}$ regulation (6-8). Deficient CFTR anion transport, and thereby reduced chloride and bicarbonate concentrations at the airway surface, leads to decreased mucus clearance, copious airway obstruction, and loss of lung function (1). Bicarbonate concentration, in particular, has been implicated as crucial to promoting appropriate mucin unfolding and normal mucus properties (9-12). Without bicarbonate, mucus release from both goblet cells and airway submucosal glands is impaired $(10,12-14)$. In the setting of abnormal mucus, hyperosmotic periciliary layer (PCL) depletion also contributes to mucus stasis $(15,16)$.

The mechanisms underlying the evolution of abnormal mucus and its clearance in $\mathrm{CF}$, as well as effective measures by which to treat the resultant mucostasis, remain unknown. In vivo studies of the CF mucus abnormality have been limited, as the CF mouse models do not accurately replicate the muco-obstructive lung disease characteristic of human patients with CF $(17,18)$. While significant insights have arisen from the CF pig and ferret models (19-24), particularly regarding mucus secretion and release at the onset of disease $(13,25)$, these models are less amenable for longitudinal studies to characterize how CF pathogenesis ensues over time. These limitations have led us to develop a $\mathrm{Cftr}^{-1-}$ rat (here referred to as the CFTR 
$\mathrm{KO}$ rat), which enables experiments out to at least 6 months of age and provides a model appropriate for therapeutic intervention coupled with mechanistic studies as the disease evolves. Preliminary characterization demonstrated completely absent CFTR expression and function in all tissues, including the airways, combined with initial events that characterize the CF lung, namely a dehydrated airway surface (26). Additionally, the rat expresses a higher number of submucosal glands in the upper airways, especially tracheae and bronchi (27), a feature that has been shown to be important to human CF disease (28-33).

In the present study, we combine traditional methods with 1-micron resolution optical coherence tomography $(\mu \mathrm{OCT})$ to show that airway submucosal glands are developmentally delayed in the CFTR $\mathrm{KO}$ rat but are required for the development of an abnormal mucus defect. Neither hyperacidic $\mathrm{pH}$ nor dehydration of the airway surface can impart mucus stasis unless gland secretions are mature, lending a crucial insight into the role of submucosal glands in the development of CF airway disease. Further, gland secretions are particularly sensitive to changes in bicarbonate concentration, but not to changes in $\mathrm{pH}$, for the initiation of delayed mucus clearance. This is a finding with important therapeutic indications, as mucus clearance is an important component of host defense and effective CF treatment.

\section{Results}

Submucosal gland development is delayed in the CFTR KO rat trachea. The phenotype of CFTR KO rats at weaning was recently described (26). At 4-6 weeks of age, overall tracheal morphology appeared similar between $\mathrm{KO}$ and WT rats, including the number and intensity of mucus-producing cells in the trachea. As rats aged, submucosal glands in the trachea became more apparent in KO rats and demonstrated submucosal gland duct plugging by 6 months, features absent in WT rats (Figure 1A). Morphometric measurements of WT and KO tracheal histology images indicated that submucosal gland area was diminished in $\mathrm{KO}$ rats at weaning but became greater than WT by 6 months of age $\left(0.172 \pm 0.3 \mathrm{~mm}^{2}\right.$ WT vs. $0.244 \pm 0.04 \mathrm{~mm}^{2} \mathrm{KO}, P<0.05$, Figure 1B). Correspondingly, the area of intracellular mucus staining became larger in KO rats compared with that in WT rats by 6 months of age (Figure 1C), even though KO rats remained smaller overall compared with their WT littermates (Supplemental Figure 1; supplemental material available online with this article; https://doi.org/10.1172/jci.insight.97199DS1). Although male rats of both genotypes grow larger than females of the same age, there was no detectable sex-related difference in histomorphometry. To assess the function of tracheal submucosal glands and the effect of their secretions on the airway surface at 1,3 , and 6 months of age, tracheae were imaged under $\mu \mathrm{OCT}$ as previously described $(22,24,26,34)$ and the airway surface liquid (ASL) depth was measured before and after cholinergic stimulation with acetylcholine (Figure 2A). Comparison of ASL depths of KO rats to those of their age-matched WT littermates showed that ASL depths were diminished in both basal and stimulated conditions, from weaning until at least 6 months of age (WT 1 month $22.9 \pm 4.6 \mu \mathrm{m}, 6$ months $43.6 \pm 12.5 \mu \mathrm{m}$ vs. KO 1 month $6.9 \pm 0.7 \mu \mathrm{m}, 6$ month $19.5 \pm 4.8 \mu \mathrm{m} ; P<0.05$ at all ages, Figure 2 , B and C). Compared with WT tracheae, those of $\mathrm{KO}$ rats had a diminished response to cholinergic-stimulated gland secretion at 1 month of age but then exceeded the WT response by 6 months of age. The absolute change in ASL depth in acetylcholine-stimulated tissue, compared with baseline, showed that WT rat tracheae responded as expected to cholinergic stimulation $(28,31,35)$, with increasing ASL depths at all ages. In contrast, $\mathrm{KO}$ rats had an almost undetectable difference in ASL depth upon cholinergic stimulation at 1 month of age, whereas fluid secretion in response to acetylcholine was greater by 3 and 6 months (Figure 2, A and D). When analyzed as percentage change over baseline, WT rats approximately doubled the volume on the surface at 1 month of age and increased the volume by about $50 \%$ at later time points (Figure 2E). KO rats, however, exhibited a minimal volume increase at 1 month but almost doubled ASL depth at 3 and 6 months of age (KO 1 month, $-1.2 \% \pm 0.1 \%, 3$ months $143.3 \% \pm 41 \%$, 6 months $95.3 \%$ $\pm 12.2 \%$ ). These data suggest that maturation of the submucosal glands in the trachea of the KO rat is delayed, not only in size but also in function compared with their WT littermates; additionally, eventually stimulated secretion response in $\mathrm{KO}$ rats exceeded that of normal rats.

Absence of CFTR alters airway surface properties of the KO rat. KO rat tracheae exhibited characteristics of the airway that have been reported in other models of $\mathrm{CF}$. The $\mathrm{pH}$ of the airway surface, measured by a small-volume $\mathrm{pH}$ probe, was hyperacidic compared with WT littermates, at all ages tested (Figure 3A). While the $\mathrm{pH}$ of both the WT and the $\mathrm{KO}$ tracheae increased slightly following cholinergic stimulation (by $0.24 \pm 0.009$ and $\mathrm{pH} 0.36 \pm 0.007 \mathrm{pH}$ units, WT and $\mathrm{KO}$, respectively), the $\mathrm{pH}$ of $\mathrm{KO}$ tracheal surface remained more acidic than that of the WT tracheal surface (Figure $3 \mathrm{~B}$ ). The solid content of the mucus, 
A
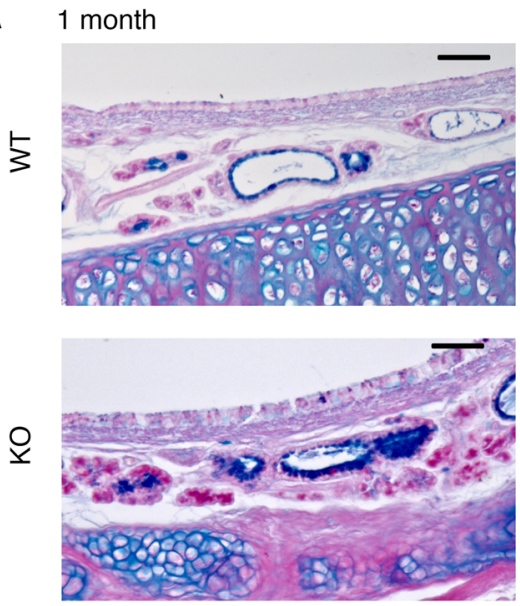

B

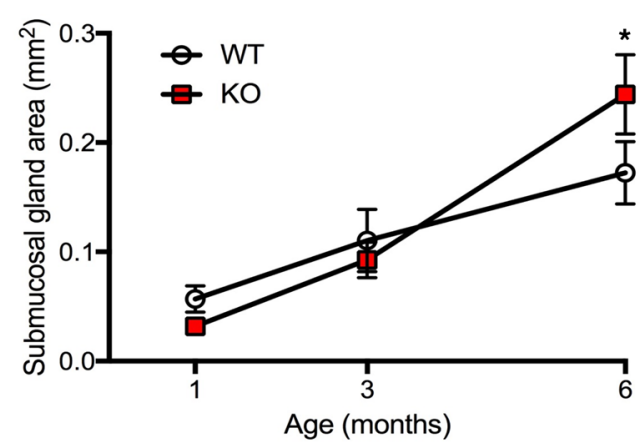

3 month
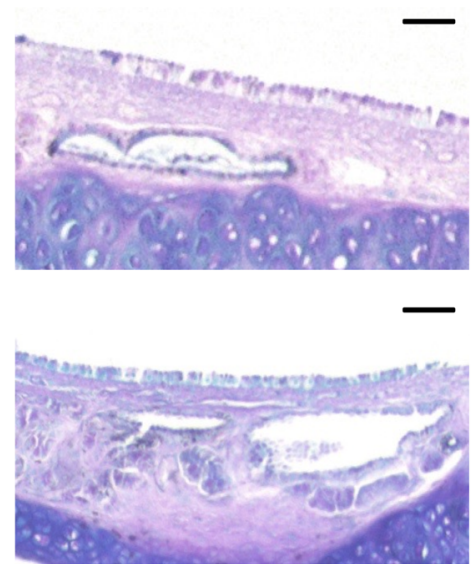

6 month
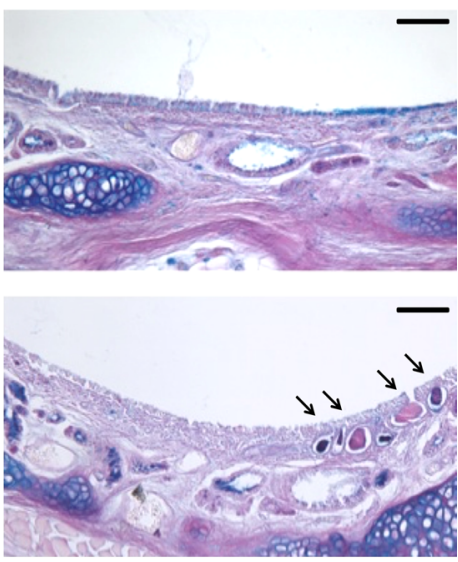

C

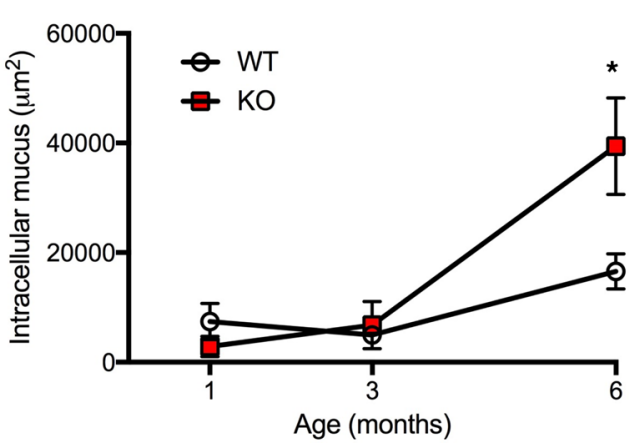

Figure 1. Tracheal gland development. (A) Representative images of Alcian blue-periodic acid-Schiff-stained (AB-PAS-stained) tracheal sections from 1-, 3-, and 6-month-old WT and KO rats. Arrows indicate mucus plugging in the gland ducts in the 6-month-old KO trachea. Scale bar: 50 mm. (B) Submucosal gland area measurements in each age and genotype. (C) Submucosal gland intracellular mucus measurements from each age and genotype. Data are shown as mean \pm SEM. Data were analyzed by 2 -way ANOVA. ${ }^{*} P<0.05$. Data in $\mathbf{A}$ are representative of 3 independent studies. Data in $\mathbf{B}$ and $\mathbf{C}$ are from 6 animals/group for each panel.

which has been reported to be increased in the CF airway $(16,36-38)$, was also measured in both WT and $\mathrm{KO}$ rat tracheae as the rats aged. The percentage of solid content of the airway mucus layer under basal conditions was increased significantly in $\mathrm{KO}$ rats at all ages evaluated (WT 1 month, 2.31\% $\pm 3.8 \%, 3$ months $2.96 \% \pm 0.6 \%, 6$ months $0.84 \% \pm 0.1 \%$ vs. KO 1 month $13.31 \% \pm 0.5 \%, 3$ months $9.93 \% \pm 0.6 \%$, 6 months $10.51 \% \pm 1.6 \%, P<0.001$ at all ages, Figure 3C). This result was consistent with reduced ASL depth and reflective of significant dehydration (Figure 2, A and B), indicating that the $\mathrm{KO}$ rat exhibits both characteristics (dehydration and abnormal $\mathrm{pH}$ ) postulated to drive $\mathrm{CF}$ pathogenesis.

Nevertheless, the effects of aging and gland secretion on this compartment of the airway surface was more complicated than the initial steady-state condition. At a young age, cholinergic stimulation resulted in reduced solid content in the $\mathrm{KO}$ tracheae, while the 3- and 6-month-old $\mathrm{KO}$ rat tracheae produced an increased percentage of solid content in response to acetylcholine (Figure 3D). Examining these data as a change from baseline after cholinergic stimulation demonstrated that the 1-month $\mathrm{KO}$ condition is unique; this is the only age at which the solid content percentage of the mucus decreases after treatment with acetylcholine (Figure 3E). $\mu \mathrm{OCT}$-based PCL depth measurements of the same animals showed that this layer was collapsed under basal conditions at 1 month, indicating local dehydration in $\mathrm{KO}$ rats compared with WT at baseline (Figure 3F), but, as with the percentage of solids, the effects of gland maturation made this more complicated. With stimulation of mucus secretion by acetylcholine treatment, PCL depths were greater in the KO than the WT rat at 1 month of age $(4.46 \pm 0.5 \mu \mathrm{m}$ WT vs. $5.45 \pm 4.2 \mu \mathrm{m} \mathrm{KO}, P<$ $0.01)$, no different at 3 months $(4.56 \pm 0.2 \mu \mathrm{m}$ WT vs. $4.05 \pm 0.4 \mu \mathrm{m} \mathrm{KO})$, and ultimately demonstrated PCL collapse at 6 months of age $(4.10 \pm 0.2 \mu \mathrm{m}$ WT vs. $3.53 \pm 0.2 \mu \mathrm{m} \mathrm{KO}$, Figure $3 \mathrm{G})$. This differential response indicates that although PCL depletion is present at 1 month, gland stimulation is principally aqueous at this age, which reduces solid content and restores the local PCL depth after induction. These 
A WT 1 month
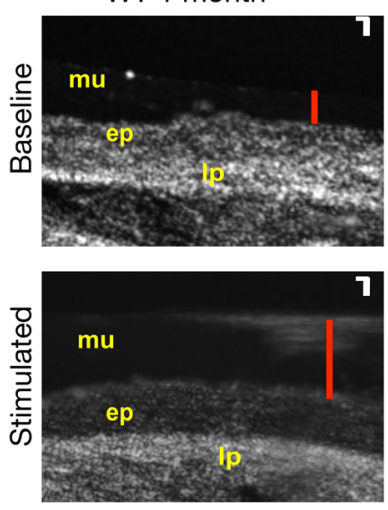

B

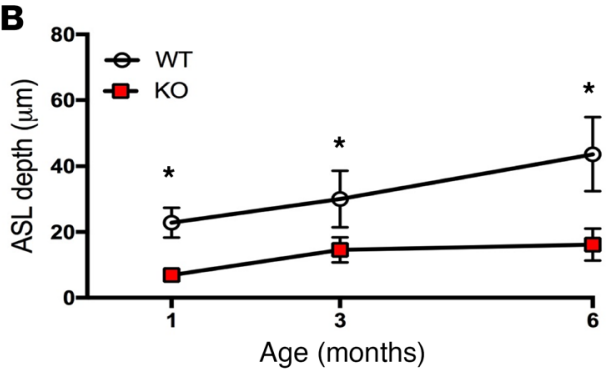

D

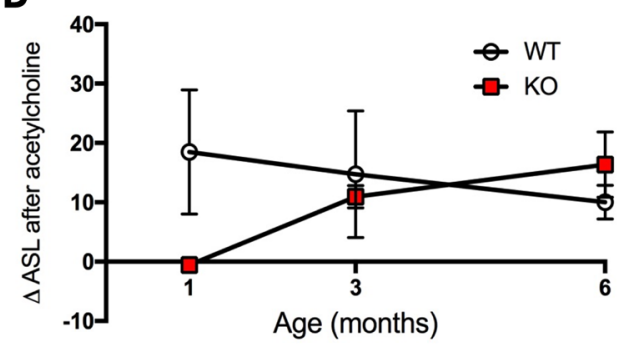

KO 1 month
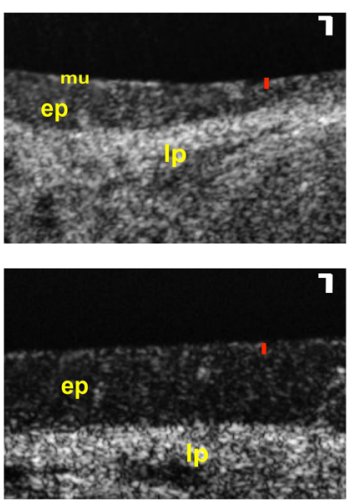

C
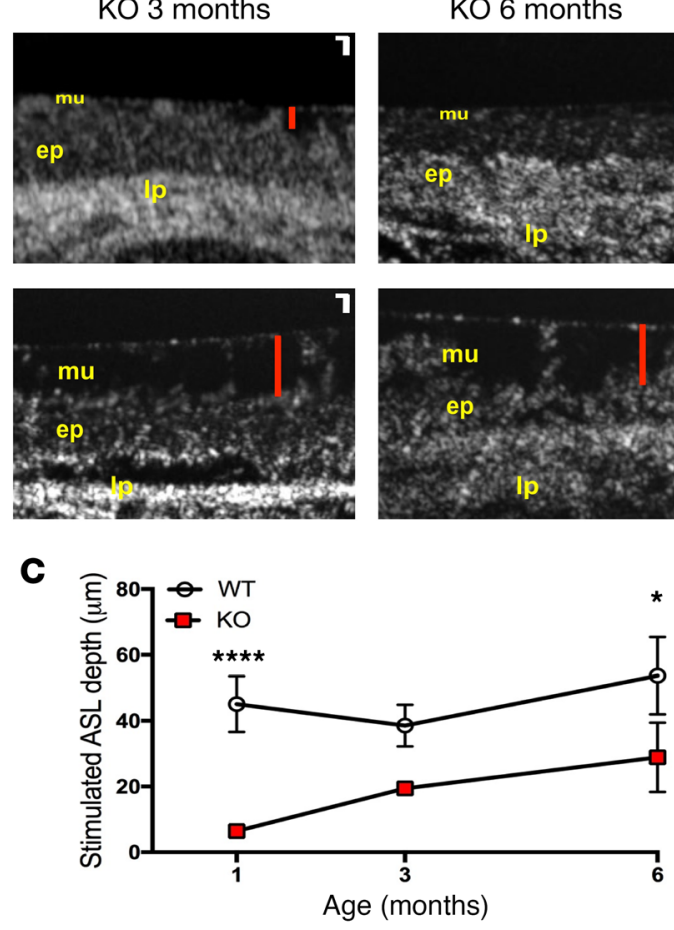

E

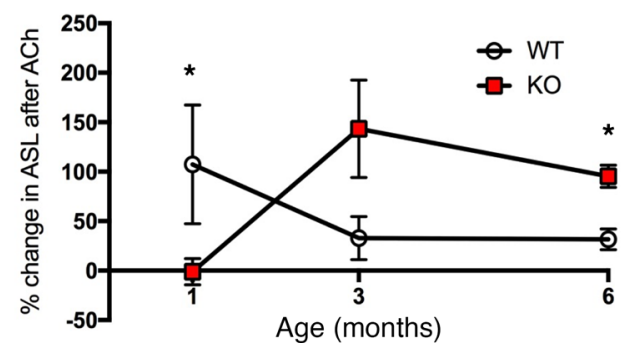

Figure 2. Airway surface liquid depletion throughout development. (A) Representative $\mu$ OCT images of WT and KO rat tracheae at baseline conditions as well as treated with acetylcholine to stimulate mucus secretion, depicting the epithelial layer (ep), mucus layer (mu), lamina propria (Ip), and airway surface liquid (ASL) depth (red bar). White bar: $10 \mu \mathrm{m}$ (in 2 dimensions). Raw images were acquired at an $0.7 \mu \mathrm{M}$ resolution. Quantification of the images yields measurements of ASL depth at baseline (B) and after cholinergic stimulation (C) as well as the absolute change (D) and percentage change (E) in ASL after acetylcholine stimulation. Data are shown as mean \pm SEM. Data were analyzed by 2 -way ANOVA. ${ }^{*} P<0.05,{ }^{* * *} P<0.0001$. Data in $\mathbf{A}$ are representative of $\mathbf{3}$ independent studies. Data in B-E are from 6 animals/group.

data further suggest that as airway submucosal glands develop over time, including maturation of the gland secretions themselves, increased solid content is delivered to the surface, contributing to PCL collapse, probably through increased osmotic force $(16,39)$ induced by elevated solid content.

Altered mucus transport and viscoelastic properties develop as the CFTR KO rat ages. Next, we evaluated the effect of changes in PCL depth, solid content, and $\mathrm{pH}$ induced by gland expression and stimulation on mucus transport to assess their effect on epithelial function and host defense. By 6 months of age, the KO rat expressed increased mucus secretion and accumulation of mucus in the small airways (area 0.5-1.5 $\mathrm{mm}$ ), coupled with an increase in the size of goblet cells, despite a similar proportion of goblet cell numbers (Supplemental Figure 2); nevertheless, as opposed to larger CF animals, there was not consistent evidence of complete occlusion of the small airways with mucus or an increase in the incidence of spontaneous infection. However, detailed investigation of the mucus properties as the rat aged revealed abnormalities. The same $\mu \mathrm{OCT}$ images that yielded measurements of ASL and PCL depths were also used to calculate mucociliary transport (MCT) rates in WT and KO tracheae. At baseline, MCT rates measured in the KO tracheae were no different than those of WT littermates; however, as the rats aged, mucus transport became signifcantly delayed, culminating at age 6 months of age (Figure 4A). Moreover, following cholinergic stimula- 

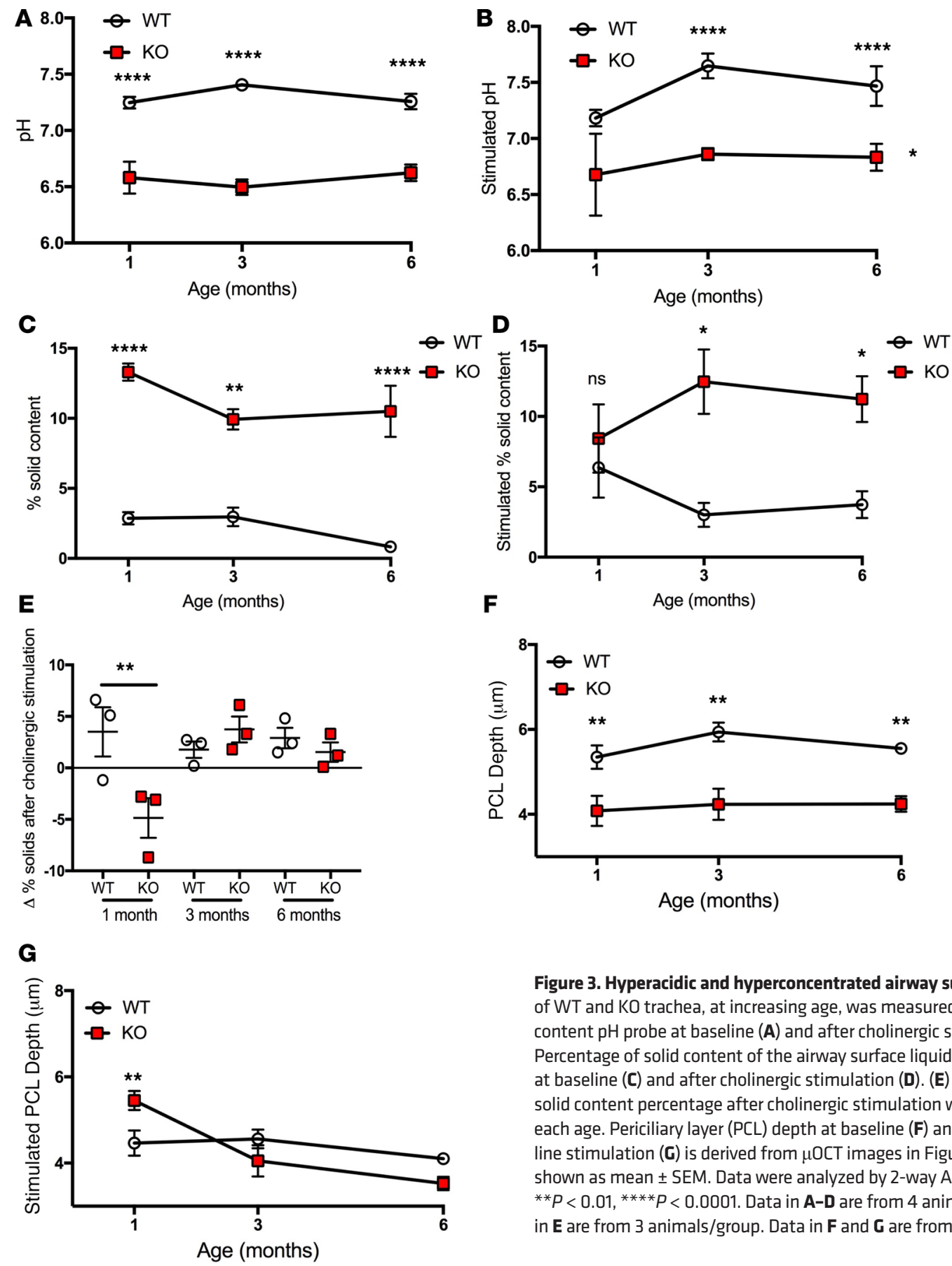

Figure 3. Hyperacidic and hyperconcentrated airway surface liquid. $\mathrm{pH}$
of $\mathrm{WT}$ and $\mathrm{KO}$ trachea, at increasing age, was measured using a solid
content $\mathrm{pH}$ probe at baseline $(\mathbf{A})$ and after cholinergic stimulation (B).
Percentage of solid content of the airway surface liquid was measured
at baseline (C) and after cholinergic stimulation $(\mathbf{D})$. (E) Change in the
solid content percentage after cholinergic stimulation was calculated for
each age. Periciliary layer (PCL) depth at baseline (F) and after acetylcho-
line stimulation $(\mathbf{C})$ is derived from $\mu 0 C \mathrm{O}$ images in Figure $2 \mathrm{~A}$. Data are
shown as mean \pm SEM. Data were analyzed by 2 -way ANOVA. ${ }^{*} P<0.05$,
${ }^{* *} P<0.01,{ }^{* * *} P<0.0001$. Data in $\mathbf{A}-\mathbf{D}$ are from 4 animals/group. Data
in $\mathbf{E}$ are from 3 animals/group. Data in $\mathbf{F}$ and $\mathbf{G}$ are from 6 animals/group.

tion, MCT rates were preserved in 1-month-old $\mathrm{KO}$ rats but were dramatically lower in the $\mathrm{KO}$ tracheae by even 3 months of age (Figure 4B). Particle-tracking microrheology, a robust technique that correlates highly to other methods of viscosity measurement, such as fluorescence recovery after photobleaching $(24,40,41)$, was used to calculate effective viscosity of the mucus on the surface of the trachea in situ. Representative tracings indicate the Brownian motion of particles in the mucus of the defined condition and demonstrate the constraints on the movement of particles in more viscous mucus (Figure 4, E-G). Under baseline conditions at 1 month of age, effective viscosity was no different in $\mathrm{KO}$ tracheae compared with WT tracheae $(1.76 \pm 1.0 \mathrm{cP}$ WT vs. $1.90 \pm 1.7 \mathrm{cP} \mathrm{KO}$, Figure $4 \mathrm{C})$. At 3 months, effective viscosity of $\mathrm{KO}$ airway mucus increased slightly but was still no different than that of WT airway mucus (5.12 $\pm 1.0 \mathrm{cP}$ WT vs. $10.72 \pm 2.7$ cP KO, Figure 4C). By 6 months of age, KO tracheal mucus was 20 -fold more viscous compared with WT littermates $(2.91 \pm 0.9 \mathrm{cP}$ WT vs. $65.09 \pm 3.6 \mathrm{cP} \mathrm{KO}, P<0.05$, Figure $4 \mathrm{C})$, consistent with delayed transport 


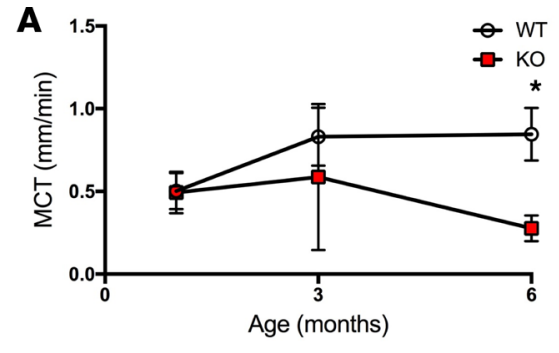

C

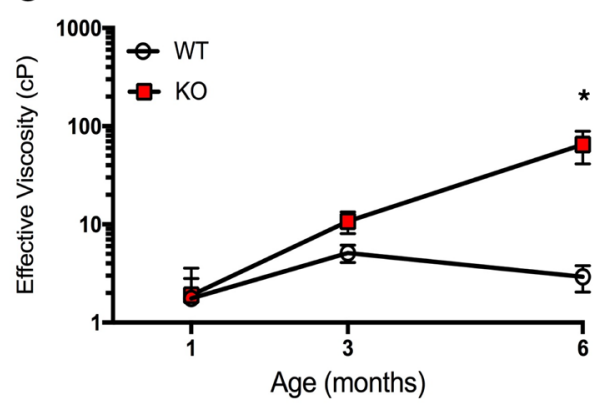

B

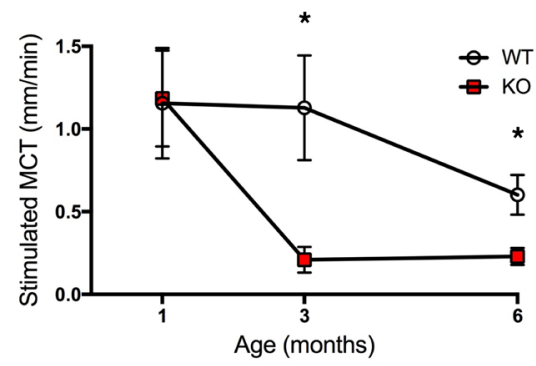

D

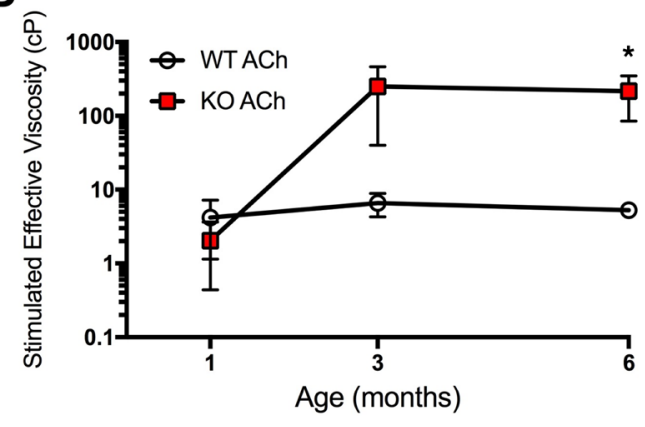

E
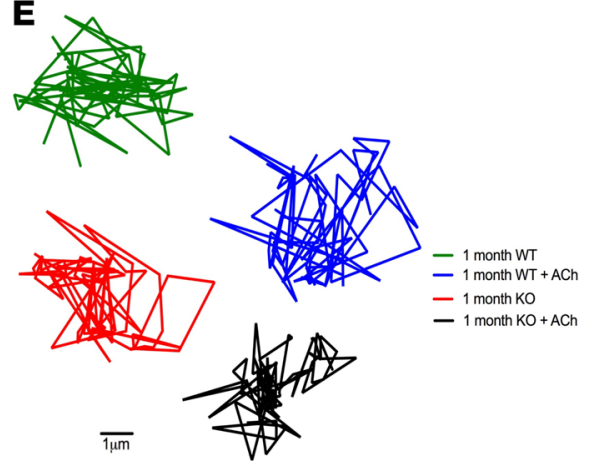

$\mathbf{F}$

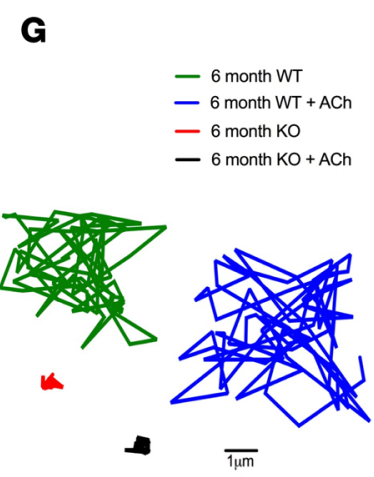

Figure 4. Altered mucus transport and viscosity. Mucociliary transport (MCT) rates at baseline conditions (A) and after stimulation with acetylcholine (B). Particle-tracking microrheology (PTM) was used to determine the effective viscosity of mucus at baseline conditions (C), which were $0.06 \mathrm{~Hz}$, and after stimulation with acetylcholine (D). Representative tracks of beads in mucus of each condition at 1 month (E), 3 months (F), and 6 months (G) of age. Scale bar: 1 $\mu \mathrm{m} .{ }^{*} P<0.05$. Data were analyzed by 2 -way ANOVA. Data in $\mathbf{A}-\mathbf{D}$ are from 6 animals/group. Data in $\mathbf{F}$ and $\mathbf{G}$ are representative of 3 independent studies.

at this age. In the stimulated conditions, airway mucus in the $\mathrm{KO}$ rat is more viscous by 3 months of age (Figure 4D), corresponding to the results seen in MCT rate analysis. Overall, these studies demonstrate that while PCL depth, solid content, and $\mathrm{pH}$ are affected at baseline in $\mathrm{CF}$ at all ages, they do not contribute to delayed MCT rates until the glands mature, when stimulated PCL collapse and increased mucus viscosity occur in response to secretion of elevated mucus solid content. Thus, mature gland secretion is required to manifest the $\mathrm{CF}$ defect primed by airway dehydration and bicarbonate deficiency.

Appropriate mucus properties are dependent on bicarbonate at the surface of the airway. Previous studies, from our laboratory and others $(12,24,42)$, have shown that bicarbonate secretion through the CFTR is criti$\mathrm{cal}$ for appropriate mucus transport. To determine whether this was true in the KO rat model and to test the relationship of this finding to mucus hydration, we conducted the same imaging experiments on WT tracheae in the presence and absence of bicarbonate and compared these to KO tracheae. In the absence of bicarbonate, 3-month-old WT tracheae exhibited reduced $\mathrm{pH}$ of the airway surface, similar to the KO tracheae (Figure 5A). Interestingly, the lack of bicarbonate transport did not change the solid content percentage (Figure 5B) or PCL depth (Figure 5C) in the 3-month-old WT rat tracheae; this is similar to the effects seen previously in pig tracheae treated with bicarbonate transport inhibitors $(24,28,42,43)$ and is consistent at 1 and 6 months of age (data not shown). In contrast, the effects of absent bicarbonate on MCT rates recapitulated diminished transport in KO tracheae but only after gland maturation; in 

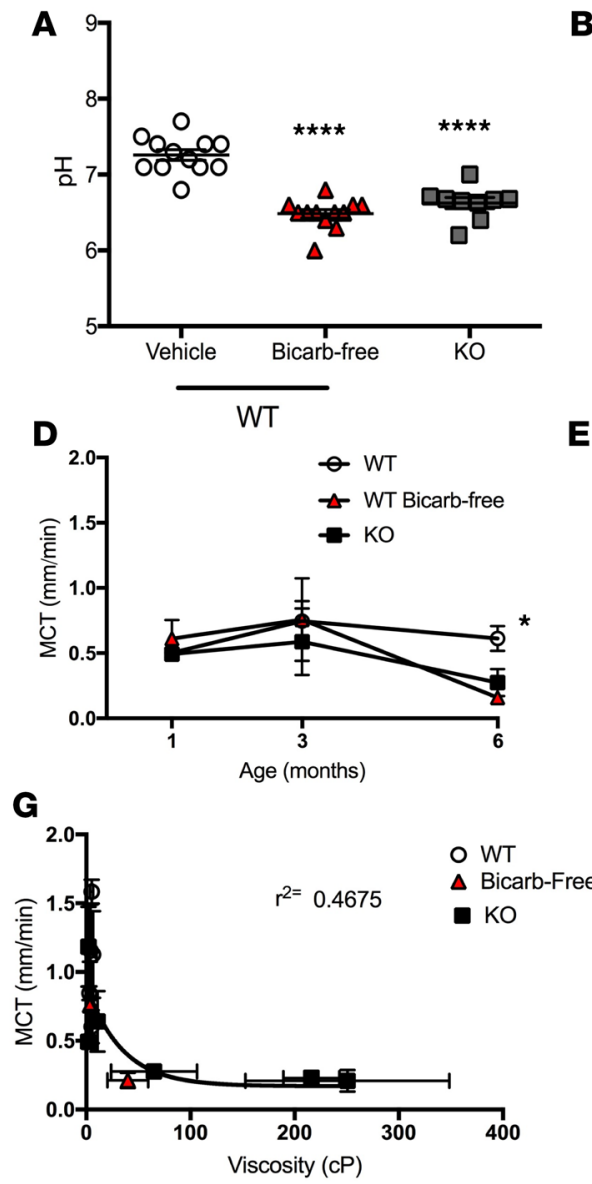

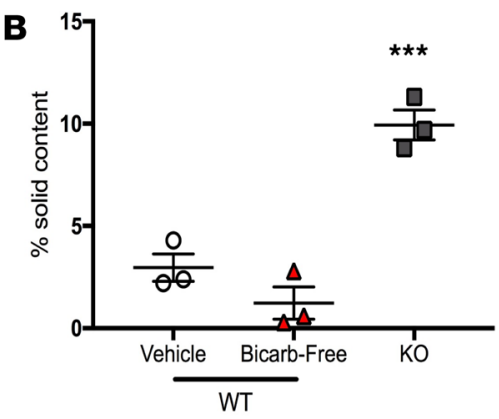

E

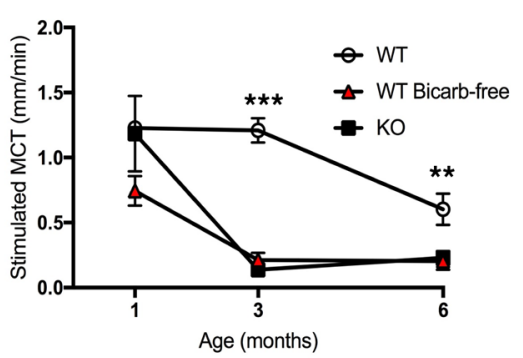

H

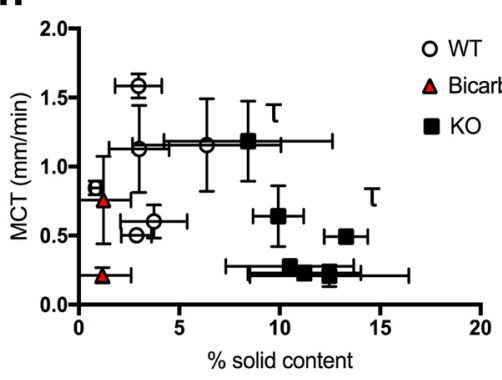

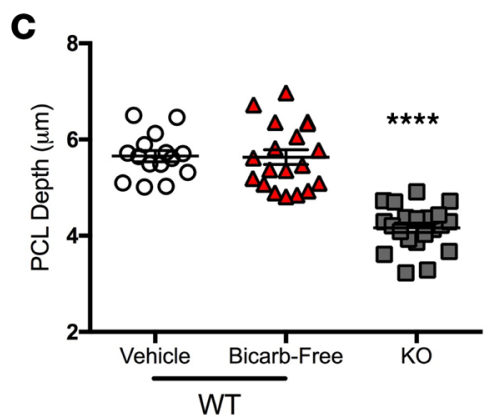

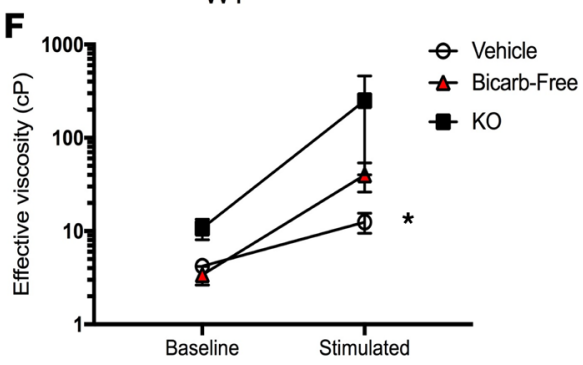

I

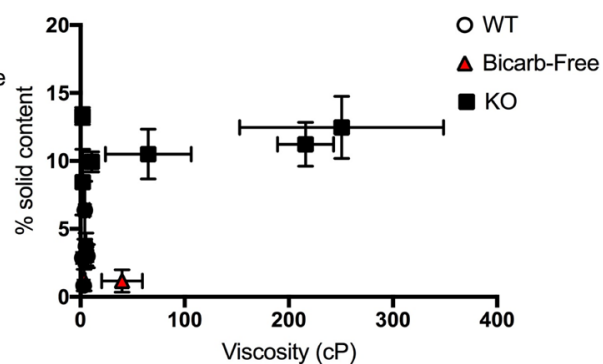

Figure 5. Hyperacidic airway surface liquid and altered mucus are bicarbonate dependent. $\mathrm{pH}$ of 3-month-old WT tracheae in normal conditions and in bicarbonate-free conditions compared with $\mathrm{KO}$ tracheae, was measured using a solid content pH probe (A). Solid content of the airway surface mucus in 3-month-old WT trachea under normal and bicarbonate-free conditions was also compared with KO trachea (B). Periciliary layer (PCL) depths of WT tracheae in normal conditions and in bicarbonate-free conditions, compared with KO trachea, obtained from $\mu$ OCT imaging (C). $\mu$ OCT imaging also yields mucociliary transport (MCT) rates at baseline (D) and after cholinergic stimulation (E). Particle-tracking microrheology (PTM) yields effective viscosity of WT tracheae in the presence and absence of bicarbonate, compared with KO (F). MCT rates compared with effective viscosity indicate a strong relationship between viscosity of the mucus and the rate at which it is transported across the trachea (G). However, MCT rates compared with the solid content percentage (1-month $\mathrm{KO}$ conditions marked by $\tau$ ) $(\mathbf{H})$ and the solid content percentage compared with viscosity (I) do not correlate in a statistically significant manner. Data are shown as mean $\pm \mathrm{SEM}$. ${ }^{*} P<0.05,{ }^{* *} P<0.01,{ }^{* * *} P<0.001,{ }^{* * * *} P<0.0001$. Data were analyzed by (A-C) 1-way ANOVA and (D-F) 2-way ANOVA. Data in $\mathbf{A}$ depict 3 regions of interest (ROls) from 4 animals/group. Data in $\mathbf{B}$ are from $\mathbf{3}$ animals/group. Data in $\mathbf{C}$ are 4 ROIs from 6 animals/group. Data in $\mathbf{D}-\mathbf{I}$ are from 6 animals/group.

bicarbonate-depleted tracheae, MCT rates were reduced at 6 months under baseline conditions (Figure 5D) and also at 3 and 6 months in cholinergically stimulated conditions (Figure 5E). Elevated effective viscosity of the airway surface mucus was also dependent on bicarbonate concentrations; 3-month-old WT tracheae, which were incubated on bicarbonate-free media and were cholinergically stimulated, had more viscous mucus on the airway surface (Figure $5 \mathrm{~F}$ ).

A comparison of $\mathrm{MCT}$ rates with corresponding effective viscosity values showed a correlation between the two characteristics $\left(r^{2}=0.468, P<0.05\right.$, Figure $\left.5 \mathrm{G}\right)$. There was a sharp decrease in MCT rates when viscosity values reached approximately $50 \mathrm{cP}$. This suggests that there is a threshold of mucus viscosity beyond which the airway is no longer able to transport effectively. In comparison, the correlation between MCT rates and the solids percentage was weak (Figure $5 \mathrm{H}$ ) as was the correlation between the solids percentage and viscosity (Figure 5I), indicating that solid content does not predict MCT rate or mucus viscosity in the CF airway in the absence of considering the maturity and quality of gland secretions. This comparison also showed that blockade of bicarbonate transport could replicate the effects on mucus viscosity and delayed transport seen in the KO rat tracheae, whereas maturation of the glands was needed to invoke PCL collapse. This indicates that bicarbonate-deficient 
A

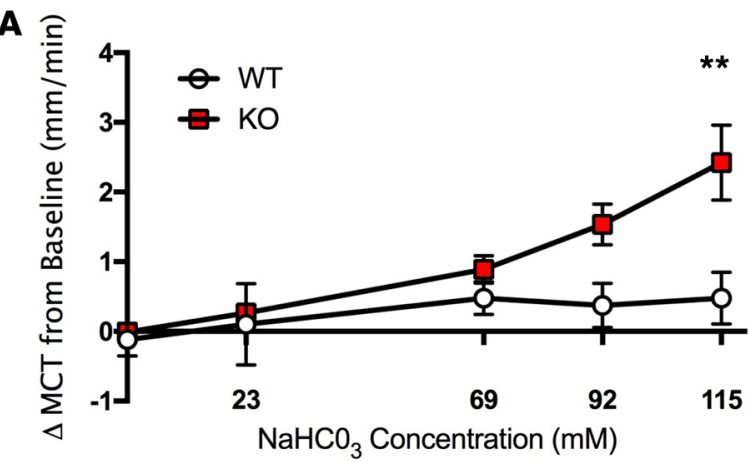

C

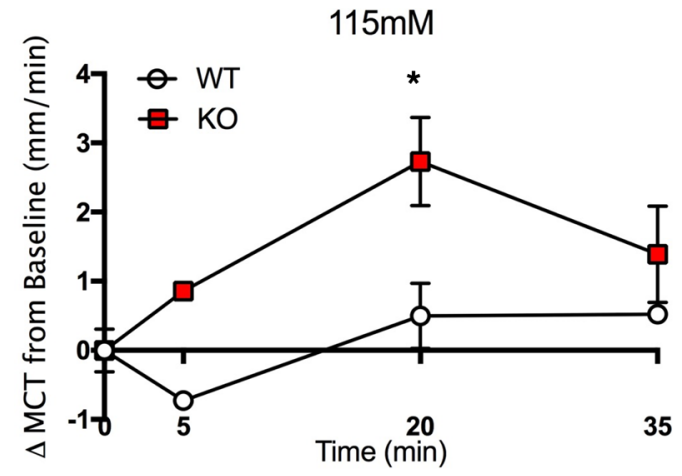

B

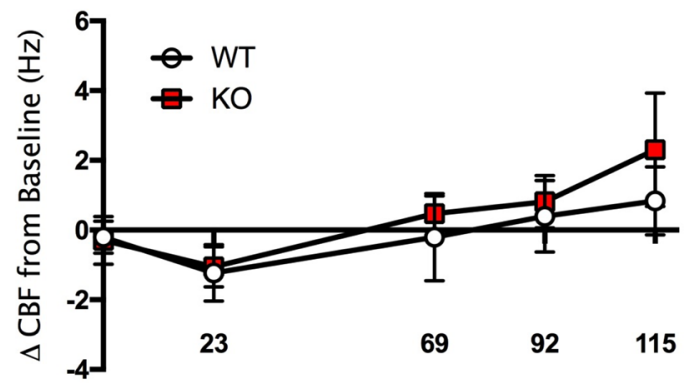

$\mathrm{NaHCO}_{3}$ Concentration (mM)

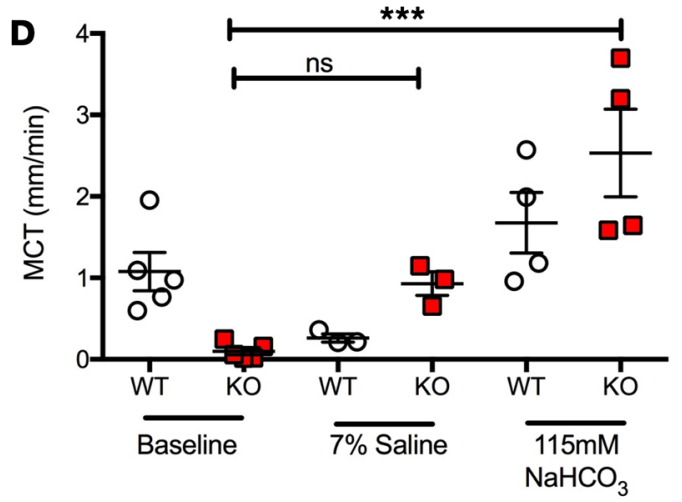

Figure 6. MCT rate recovery with apical treatment with sodium bicarbonate. Mucociliary transport (MCT) rates compared with baseline of 6-month-old WT and KO tracheae treated with increasing concentrations of sodium bicarbonate (A) and corresponding ciliary beat frequency (CBF) (B). MCT rates compared with baseline in 6-month-old WT and KO tracheae treated with hypertonic saline or $115 \mathrm{mM}$ sodium bicarbonate (C). MCT rates compared with baseline in 6-month-old WT and KO tracheae after addition of hypertonic saline or $115 \mathrm{mM}$ sodium bicarbonate (D). ${ }^{*} P<0.05,{ }^{* *} P<0.01 . n=4-6$ animals/ group. Data were analyzed by (A-C) 2-way ANOVA and (D) 1-way ANOVA. Data are from 4 animals/group.

mucus exhibits abnormal properties independent of the deleterious effects of airway dehydration. It also indicated that the bicarbonate deficiency in the CF airway may be a therapeutic target to restore appropriate mucus transport.

Addition of bicarbonate restores normal mucus transport in the CFTR KO rat. Because bicarbonate concentrations were important to the maintenance of appropriate viscosity and mucus transport, we hypothesized that replacement of bicarbonate on the airway surface would restore aberrant mucus transport in the KO rat tracheae, a concept proposed for CF therapy but not yet tested in vivo (44). These experiments also provided an opportunity to test an intervention independent of airway hydration to define the importance of bicarbonate balance on MCT rates. In tracheae from 6-month-old WT and $\mathrm{KO}$ rats, we conducted $\mu \mathrm{OCT}$ imaging at baseline and following addition of sodium bicarbonate at concentrations of $23 \mathrm{mM}, 69 \mathrm{mM}, 92 \mathrm{mM}$, and $115 \mathrm{mM}$ to the apical surface of tracheae ex vivo or sodium chloride at $7 \%$ (hypertonic saline) as a control for added volume and to compare with a widely used CF therapy (45-47). MCT rates were measured at 5, 20, and 35 minutes following addition of each solution. Compared with baseline, MCT rates in KO rat tracheae increased with each additional concentration of sodium bicarbonate, while MCT rates were unchanged from baseline in WT rat tracheae (Figure 6A). Ciliary beat frequency did not significantly change from baseline in either WT or KO rat tracheae (Figure 6B), indicating that ciliary dynamics were not principally responsible for the increased transport. Hypertonic saline addition to the apical surface also resulted in a significant increase in MCT rate from baseline and was preferentially effective in KO tracheae, but efficacy was less than that when $115 \mathrm{mM}$ bicarbonate was applied $(2.53 \pm 0.5 \mathrm{~mm} / \mathrm{min} \mathrm{KO}$ plus bicarbonate, $0.92 \pm 0.1 \mathrm{~mm} / \mathrm{min} \mathrm{KO}$ plus hypertonic saline, vs. $0.09 \pm 0.04 \mathrm{~mm} / \mathrm{min}$ KO baseline, $P<0.001$ bicarbonate vs. baseline, Figure 6D). Despite substantial efficacy of sodium bicarbonate on MCT rates in KO tracheae, the improvement was transient; the peak effect occurred at 20 minutes after addition and returned to baseline by 35 minutes after addition (Figure 6C). This finding is consistent with the short half-life of bicarbonate at the surface of the airway, even at concentrations much higher than physiologic, likely due to ubiquitous expression of carbonic anhydrase $(48,49)$. 
A

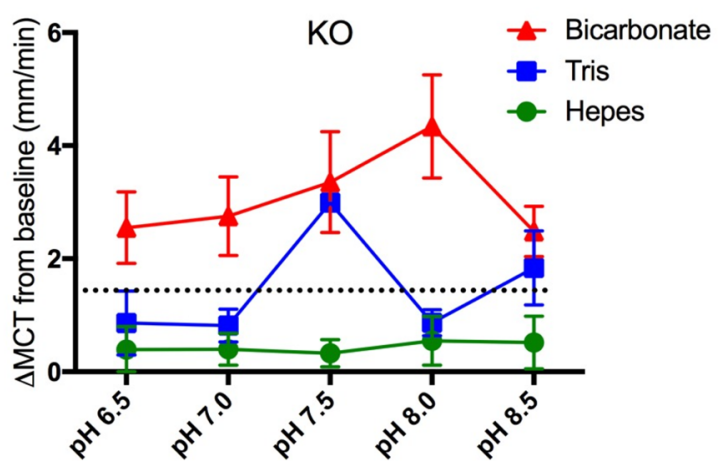

C

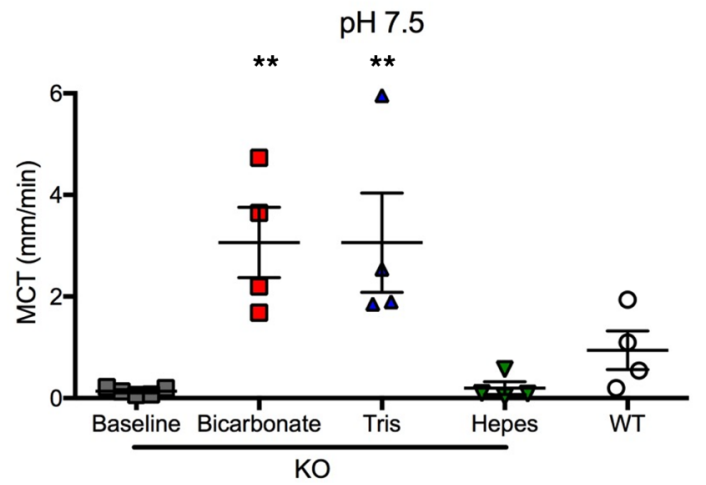

B

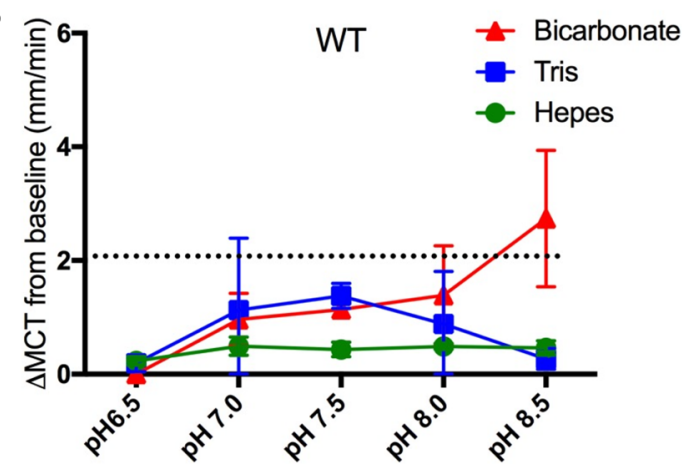

Figure 7. MCT normalization requires $\mathrm{pH}$-dependent counterion equilibrium. Mucociliary transport (MCT) rates compared with baseline when treated with sodium bicarbonate, Tris, or HEPES buffered to a $\mathrm{pH}$ of between 6.5 and 8.5 in 6-month-old KO (A) and WT (B) tracheae. Comparison of MCT rates in 6-month-old KO tracheae at baseline or treated with different solutions buffered to $\mathrm{pH} 7.5$ compared with WT tracheae at baseline (C). ${ }^{*} P<0.05$. The dotted line in A and $\mathbf{B}$ indicates $P<0.05$ by 2-way ANOVA. $n=4-6$ animals/group. Data in $\mathbf{C}$ were analyzed by was analyzed by 1-way ANOVA. Data are from 4 animals/group.

The effect of bicarbonate addition on MCT rate could be due to altered bicarbonate concentration, alkalinization of the airway $\mathrm{pH}$, or a combination of the two, since the solutions of sodium bicarbonate used here were not $\mathrm{pH}$ adjusted. To distinguish these mechanisms, we tested solutions of $115 \mathrm{mM}$ sodium bicarbonate, $50 \mathrm{mM}$ Tris, and $10 \mathrm{mM}$ HEPES and buffered these solutions to $\mathrm{pH} 6.5,7.0,7.5$, 8.0, and 8.5. As before, solutions were added to the apical surface of 6-month-old WT and KO rat tracheae, and MCT rates 20 minutes after addition were compared with baseline MCT rates. We found that sodium bicarbonate added at any $\mathrm{pH}$ increased MCT rates significantly above baseline in $\mathrm{KO}$ tracheae. By contrast, addition of Tris, which has the ability to donate or accept hydrogen ions as necessary, only increased MCT rates when buffered to $\mathrm{pH} 7.5$, which approximated the normal WT $\mathrm{pH}$ (approximately 7.2). In contrast, addition of HEPES solution, which, as a zwitterion, has less ability to donate or accept hydrogen ions and therefore is less interactive with its environment, did not alter MCT rates in the KO tracheae from baseline at any $\mathrm{pH}$ tested (Figure 7A). These same solutions added to the apical surface of 6-month-old WT tracheae had minimal and nonsignificant effects on MCT rates (Figure 7B). In comparing absolute values of mucus transport in $\mathrm{KO}$ tracheae at baseline and 20 minutes after each solution at $\mathrm{pH} \mathrm{7.5,} \mathrm{both} \mathrm{sodium} \mathrm{bicarbonate} \mathrm{and} \mathrm{Tris} \mathrm{solutions} \mathrm{restored} \mathrm{mucus} \mathrm{transport} \mathrm{in} \mathrm{the} \mathrm{KO} \mathrm{rat} \mathrm{tracheae,}$ exceeding MCT rates beyond that observed in the WT tracheae at baseline $(3.35 \pm 0.9 \mathrm{~mm} / \mathrm{min} \mathrm{KO}$ plus bicarbonate, $3.06 \pm 0.9 \mathrm{~mm} / \mathrm{min} \mathrm{KO}$ plus Tris, compared with $0.095 \pm 0.02 \mathrm{~mm} / \mathrm{min} \mathrm{KO}$ baseline, $P<$ 0.001 , and $0.69 \pm 0.2 \mathrm{~mm} / \mathrm{min}$ WT baseline, Figure 7C). These data indicate that, although the abnormal MCT becomes increasingly severe in the maturing KO rat airway, the defect may be amenable to mucus-targeting therapeutics that restore $\mathrm{pH}$-dependent counterion equilibrium.

\section{Discussion}

Here for the first time to our knowledge, using complimentary methods and the ability to monitor CF rats over time, we have established a relationship between the development of submucosal glands and the onset of abnormal mucus transport and viscosity in CF. We also demonstrate that both airway dehydration and bicarbonate deficiency independently contribute to the CF mucus defect in this model. Further, we have demonstrated that aberrant mucus transport can be corrected but only by ionic exchange to normalize mucus viscosity. This findings are significant as they lend insight into fundamental aspects of mucus maturation and point to therapeutic interventions to restore mucus transport that may be independent of specific CFTR mutation and, thus, could represent a viable target in all patients with CF. 
Detailed investigations in the present study demonstrate that age-dependent changes in submucosal glands may be critical for the KO rat to develop mucus abnormalities. The mechanisms underlying the developmental delay of submucosal glands are unclear but may be related to defective CFTR secretion that manifests over time. The eventual emergence of glandular hyperplasia in the tracheae is relevant to human disease, as submucosal glands are thought to be important to airway disease development, as dense mucus secretions and dilated gland ducts were a recognized feature of the CF airways, even early in disease progression $(32,50,51)$. The fluid content of the gland secretions is heavily dependent on anion transport and is therefore highly susceptible to dysfunctional CFTR (31). The correlation of glandular hypertrophy with the appearance of the mucus defect in the rat is additional evidence to suggest that the properties of submucosal gland mucus are very important to the CF defect.

While the development of a mucus defect is an important feature of the model, the younger CFTR KO rat offers an opportunity to investigate the relative contributions of the other components of the mucosal surface (before gland maturation). Even in the absence of functional gland secretion, the 1-month-old KO rat exhibits a partial phenotype, with a dehydrated, hyperacidic airway and elevated solid content of the airway mucus; however, mucus transport and viscosity remain normal despite these features. These data suggest that both PCL depletion and hyperacidic $\mathrm{pH}$ may be necessary but are not sufficient to cause the $\mathrm{CF}$ mucus defect in the absence of mature gland secretions. Mucus secretion, likely principally through glands, is also needed to induce disease. These data may help our understanding of the progression of human CF airway disease, in which airway disease, mucus obstruction, and lung function worsen as the patient ages, as well as our understanding of progression after certain triggers, such as viral or bacterial infection or particle inhalation, when mucus secretion is enhanced (52-55).

Inadequate mucus hydration, reflected by measurement of both PCL depth and the percentage of solids, and abnormal mucus rheology have been suggested as inherent causes of the CF mucus defect $(6,7$, $11,15,56)$. These two characteristics interact heavily with one another and are difficult to parse; however, the data presented here suggest that they are not completely dependent upon one another. This is particularly evident in young $\mathrm{KO}$ rats, when cholinergic stimulation normalized PCL depth and the percentage of solids, likely through aqueous fluid secretion with few glandular solids. Likewise, the hyperacidic $\mathrm{pH}$ at the young age did not confer abnormal mucus transport. Secretions at this young age are hyperacidic and osmotically depleted but not hyperviscous, providing evidence that not all solids are created equal and that they may not all be $\mathrm{pH}$ sensitive. This is reflected in the aberrant mucus properties identified by combined evaluation of percentage of solid content and the particle-tracking microrheology methods. In 6-month-old rats, the classical CF phenotype emerges; the PCL collapse under the presence of thicker, glandular secretions is correlated to an emerging mucus transport defect and suggests that a mature mucus load imparts the osmotic pressure imbalance seen in many different models of CF $(7,15)$. The abundance of mucins secreted from the glands and the role of these to clear the airways emphasize the importance of glandular secretions and may explain why abnormalities in these secretions have such a dramatic effect by 6 months of age $(57,58)$. This effect can also be recapitulated at an intermediate age ( 3 months) when gland secretion is stimulated. Taken together, these results strongly suggest that the effects of dehydration and $\mathrm{pH}$ are necessary but require the effects of functional airway submucosal glands to cause the complete mucus defect. This may be why CF piglets and neonates are often normal early in life but progress as infectious insults mount, increasing the stimulus for mucus secretion $(59,60)$.

Indeed, these mechanisms appear to be distinct when searching for a means of correction of the mucus defect. Merely adding volume to the KO rat trachea, in the case of hypertonic saline, did result in an increase in mucus transport rates, as expected from previous studies using rehydration as a therapeutic technique (61); however the addition of bicarbonate increased transport rates to a much higher degree. By contrast, adjustment of $\mathrm{pH}$ only increased transport under specific conditions. Addition of the zwitterionic compound HEPES did not alter mucus transport at all, even at the physiologic $\mathrm{pH}$ of 7.5, perhaps because it cannot donate a counterion. In contrast, addition of the ionic Tris buffer at a normal $\mathrm{pH}$, or bicarbonate addition at any $\mathrm{pH}$ within the physiologic spectrum, increased MCT rates of CF mucus. These results provide guidance, as mucus normalization strategies are considered for CF therapy, and point out that ionic exchange may be a prerequisite (62). This mechanism may also be contributing to calcium chelation of bicarbonate, an effect that has been demonstrated in other model systems (63). It has been previously shown that both dehydrated mucus and decreased $\mathrm{pH}$ independently cause increased viscosity in the CF condition $(36,64)$, likely without altering other 
biophysical factors of the mucus, such as reduction/oxidation states (65); therefore, we hypothesize that the various means of restoration of normality tested here all will result in a reduction of mucus viscosity to more transportable range.

The data presented in this paper offer a model for study of CF airway disease progression, providing a phenotype (before gland development) that may mimic early CF lung disease as well as a state of mucus abnormality (after gland secretion matures) that may more accurately replicate later stages of CF lung disease. Further, data gleaned from these studies indicate that the mechanisms that underlie aberrant mucus movement across the epithelial surface may not fit neatly into one hypothesis but rather involve several factors. This provides a tool to assess what changes as the airway glands mature, allowing the full CF defect to emerge.

\section{Methods}

CFTR KO rat model. All experiments used SD-CFTR ${ }^{\text {tmlsage }}$ rats (Horizon Discovery), either KO rats or their littermate controls, $C F T R^{+/+}$(termed WT) rats. This rat strain was bred and genotyped as previously described (26). In brief, heterozygote $\left(\mathrm{CFTR}^{+-}\right)$males and females were paired to generate WT and KO pups, and litters remained with lactating dams until 21 days of age. WT and KO rats were maintained on a standard rodent diet, with supplemental DietGel 76A (ClearH20) and 50\% Go-LYTLEY (Braintree Laboratories Inc.) added to the water from weaning as a means to reduce mortality from gastrointestinal obstruction. CFTR KO pups have a 50\% survival rate under these nutritional conditions but remain up to $30 \%$ smaller than their WT littermates by weight. WT and KO rats were assayed at 1, 3, and 6 months of ages, and groups were split evenly between males and females.

Histology/morphometry. Tracheae and lungs were immersion fixed in $4 \%$ buffered formalin and embedded in paraffin blocks for sectioning. Sections were stained with Alcian blue-periodic acid-Schiff. Slides were imaged; submucosal gland area, intracellular mucus area, goblet cell number, goblet cell area, and intra-airway mucus area were measured morphometrically, as previously described (26).

$\mu O C T$ image acquisition and analysis. Measurements of functional microanatomic parameters in ex vivo tissue were performed using $\mu \mathrm{OCT}$, a high-speed, high-resolution microscopic reflectance imaging modality. Tracheae were excised and immediately placed on gauze soaked in F12 media, such that the apical surface of the trachea remained media free, and incubated under physiologic conditions $\left(37^{\circ} \mathrm{C}, 5 \% \mathrm{CO}_{2}\right.$, $100 \%$ humidity; using live-cell imaging incubation systems, ref. 24; Carl Zeiss). Tracheae were allowed to equilibrate for 1 hour before imaging. Following baseline image acquisition, tracheae were moved onto F12 media-soaked gauze with $100 \mu \mathrm{M}$ acetylcholine added and incubated again for 30 minutes at physiologic conditions and reimaged. We have previously described $\mu \mathrm{OCT}$ methods for interrogation of airway epithelia and quantitative image analysis, including calibration of distance and height measurements (34). Images were acquired at 5 controlled points along the tracheal length, using the cranial end as a reference. MCT rate was determined using time elapsed and distance traveled of native particulates in the mucus over multiple frames, using the pixel-to-micron conversion factor determined with image calibration. For each trachea, images were acquired at standard distances along the ventral surface, with the optical beam scanned along the longitudinal direction.

Measurement of the solid content percentage. Tracheae were prepared as above. Filter paper was cut to a size that would fit inside an open tracheae. Papers were weighed, placed on each trachea for 15 seconds, and immediately reweighed to determine mucus collection. Filters were left overnight in a dry oven for liquid evaporation and weighed again for measurement of solid content. All weights were taken using a UMX2 Ultra-microbalance (Mettler Toledo). Filter weights were subtracted, leaving wet and dry weights of the mucus, which were used to calculate the fraction that was solid content.

Particle-tracking microrheology. Particle-tracking microrheological techniques were used to measure viscosity of mucus on rat tracheae in situ. Tracheae were treated with $0.1 \%$ benzalkonium chloride (Acros Organics) for 1 hour at $37^{\circ} \mathrm{C}$ to stop ciliary beating. Polystyrene beads were used as previously described $(24,40)$. Baseline $\mu \mathrm{OCT}$ images were acquired; tracheae were incubated for 30 minutes at $37^{\circ} \mathrm{C}$ on media with $100 \mu \mathrm{M}$ acetylcholine; and then stimulated $\mu \mathrm{OCT}$ images were acquired. Images were analyzed using ImageJ (NIH) and the SpotTracker plug-in (66). Resulting particle tracks were analyzed with custom Matlab procedures to compute mean squared displacement (MSD), while subtracting spurious bulk motion common to all tracks. Dynamic viscosity was derived from MSD by application of the generalized Stokes-Einstein relation as described previously $(24,40)$. 
Transport inhibition. In some experiments, $100 \mu \mathrm{M}$ acetylcholine was added basolaterally to the F12 media for cholinergically stimulated mucus release. For experiments that blocked chloride transport, 100 $\mu \mathrm{M}$ bumetanide was added to F12 media; bicarbonate transport was inhibited with use of F12 media without sodium bicarbonate. Tracheae were incubated under all conditions for 30 minutes before $\mu \mathrm{OCT}$ imaging. Bicarbonate-free experiments were incubated in $0 \% \mathrm{CO}_{2}$.

Apical addition. For MCT rescue experiments, $5 \mu \mathrm{l}$ of each solution was added to the distal end of the apical surface of the tracheae and allowed to equilibrate before $\mu \mathrm{OCT}$ imaging at 5, 20, and 35 minutes after addition. Solutions added were sodium bicarbonate at concentrations of $23 \mathrm{mM}, 69 \mathrm{mM}, 93 \mathrm{mM}$, and $115 \mathrm{mM}$ (non-pH adjusted); 0.9\% saline (non-pH adjusted); 7\% saline (HTS, non-pH adjusted); $115 \mathrm{mM}$ sodium bicarbonate adjusted to a $\mathrm{pH}$ of $6.5,7.0,7.5,8.0$, or $8.5 ; 50 \mathrm{mM}$ Tris-buffered saline, adjusted to a $\mathrm{pH}$ of $6.5,7.0,7.5,8.0$, or 8.5; and $100 \mathrm{mM}$ HEPES, adjusted to a $\mathrm{pH}$ of $6.5,7.0,7.5,8.0$, or 8.5.

Statistics. Statistical analysis was performed with GraphPad Prism version 7.0. Inferential statistics (mean, SD, and SEM) were computed using ANOVA or 2-tailed unpaired or paired $t$ test, as appropriate. For multiple comparisons, post-hoc testing was applied only if ANOVA was significant. $P$ values of less than 0.05 were considered significant. Statistics are presented as mean \pm SEM, unless indicated otherwise. Except where noted, mean values per trachea explant are reported.

Study approval. All animal use was approved by the University of Alabama at Birmingham IACUC.

\section{Author contributions}

SEB, KLT, MVF, EJS, and SMR conceived of the experiments; SEB, CMF, KLT, AMO, and JMD performed research; SEB, CMF, KLT, and KKC analyzed the data; SEB, GJT, MVF, EJS, and SMR contributed reagents, materials, and/or analysis tools; SEB and SMR wrote the manuscript; and GJT, MVF, EJS, and SMR supervised the project.

\section{Acknowledgments}

This work was supported by the National Heart, Lung, and Blood Institute (R01HL116213, to GJT and SMR; 5T32HL105346-04, to SEB; 1K08HL131867, to SEB) and the National Institute of Diabetes and Digestive and Kidney Diseases (P30DK072482, to EJS and SMR) of the National Institutes of Health, the Cystic Fibrosis Foundation Research Development Program (R464-CF), Cystic Fibrosis Foundation Mucociliary Clearance Consortium (to GJT and SMR), the postdoctoral research fellowship (to SEB) of the Cystic Fibrosis Foundation, and the Cystic Fibrosis Research Scholars Program at Gilead Sciences (to SEB). The authors acknowledge the assistance of Dezhi Wang and the Histomorphometry and Molecular Analysis Core in the Center for Metabolic Bone Disease.

Address correspondence to: Steven M. Rowe, Department of Medicine, University of Alabama at Birmingham, MCLM 768, Birmingham, Alabama 35294-0005, USA. Phone: 205.934.9640; Email: SRowe@peds.uab.edu.

1. Rowe SM, Miller S, Sorscher EJ. Cystic fibrosis. N Engl J Med. 2005;352(19):1992-2001.

2. De Boeck K, Vermeulen F, Dupont L. The diagnosis of cystic fibrosis. Presse Med. 2017;46(6 Pt 2):e97-e108

3. Poulsen JH, Fischer H, Illek B, Machen TE. Bicarbonate conductance and pH regulatory capability of cystic fibrosis transmembrane conductance regulator. Proc Natl Acad Sci USA. 1994;91(12):5340-5344.

4. Quinton PM. Missing Cl conductance in cystic fibrosis. Am J Physiol. 1986;251(4 Pt 1):C649-C652.

5. Welsh MJ. An apical-membrane chloride channel in human tracheal epithelium. Science. 1986;232(4758):1648-1650.

6. Matsui H, et al. Evidence for periciliary liquid layer depletion, not abnormal ion composition, in the pathogenesis of cystic fibrosis airways disease. Cell. 1998;95(7):1005-1015.

7. Tarran R. Regulation of airway surface liquid volume and mucus transport by active ion transport. Proc Am Thorac Soc. 2004;1(1):42-46.

8. Pezzulo AA, et al. Reduced airway surface $\mathrm{pH}$ impairs bacterial killing in the porcine cystic fibrosis lung. Nature. 2012;487(7405):109-113.

9. Chen EY, Yang N, Quinton PM, Chin WC. A new role for bicarbonate in mucus formation. Am J Physiol Lung Cell Mol Physiol. 2010;299(4):L542-L549.

10. Garcia MA, Yang N, Quinton PM. Normal mouse intestinal mucus release requires cystic fibrosis transmembrane regulatordependent bicarbonate secretion. J Clin Invest. 2009;119(9):2613-2622.

11. Quinton PM. Cystic fibrosis: impaired bicarbonate secretion and mucoviscidosis. Lancet. 2008;372(9636):415-417.

12. Gustafsson JK, et al. Bicarbonate and functional CFTR channel are required for proper mucin secretion and link cystic fibrosis with its mucus phenotype. J Exp Med. 2012;209(7):1263-1272. 
13. Hoegger MJ, et al. Impaired mucus detachment disrupts mucociliary transport in a piglet model of cystic fibrosis. Science. 2014;345(6198):818-822.

14. Yang N, Garcia MA, Quinton PM. Normal mucus formation requires cAMP-dependent HCO3-secretion and Ca2+-mediated mucin exocytosis. J Physiol (Lond). 2013;591(18):4581-4593.

15. Button B, et al. A periciliary brush promotes the lung health by separating the mucus layer from airway epithelia. Science. 2012;337(6097):937-941.

16. Hill DB, et al. A biophysical basis for mucus solids concentration as a candidate biomarker for airways disease. PLoS ONE. 2014;9(2):e87681.

17. Wilke M, et al. Mouse models of cystic fibrosis: phenotypic analysis and research applications. J Cyst Fibros. 2011;10 Suppl 2:S152-S171.

18. Guilbault C, Saeed Z, Downey GP, Radzioch D. Cystic fibrosis mouse models. Am J Respir Cell Mol Biol. 2007;36(1):1-7.

19. Rogers CS, et al. Disruption of the CFTR gene produces a model of cystic fibrosis in newborn pigs. Science. 2008;321(5897):1837-1841.

20. Stoltz DA, et al. Cystic fibrosis pigs develop lung disease and exhibit defective bacterial eradication at birth. Sci Transl Med. 2010;2(29):29ra31.

21. Sun X, et al. Disease phenotype of a ferret CFTR-knockout model of cystic fibrosis. J Clin Invest. 2010;120(9):3149-3160.

22. Keiser NW, et al. Defective innate immunity and hyperinflammation in newborn cystic fibrosis transmembrane conductance regulator-knockout ferret lungs. Am J Respir Cell Mol Biol. 2015;52(6):683-694.

23. Keiser NW, Engelhardt JF. New animal models of cystic fibrosis: what are they teaching us? Curr Opin Pulm Med. 2011;17(6):478-483.

24. Birket SE, et al. A functional anatomic defect of the cystic fibrosis airway. Am J Respir Crit Care Med. 2014;190(4):421-432

25. Hoegger MJ, et al. Assessing mucociliary transport of single particles in vivo shows variable speed and preference for the ventral trachea in newborn pigs. Proc Natl Acad Sci USA. 2014;111(6):2355-2360.

26. Tuggle KL, et al. Characterization of defects in ion transport and tissue development in cystic fibrosis transmembrane conductance regulator (CFTR)-knockout rats. PLoS ONE. 2014;9(3):e91253.

27. Widdicombe JH, Chen LL, Sporer H, Choi HK, Pecson IS, Bastacky SJ. Distribution of tracheal and laryngeal mucous glands in some rodents and the rabbit. J Anat. 2001;198(Pt 2):207-221.

28. Inglis SK, Corboz MR, Taylor AE, Ballard ST. Effect of anion transport inhibition on mucus secretion by airway submucosal glands. Am J Physiol. 1997;272(2 Pt 1):L372-L377.

29. Sturgess J, Imrie J. Quantitative evaluation of the development of tracheal submucosal glands in infants with cystic fibrosis and control infants. Am J Pathol. 1982;106(3):303-311.

30. Verkman AS, Song Y, Thiagarajah JR. Role of airway surface liquid and submucosal glands in cystic fibrosis lung disease. Am $J$ Physiol, Cell Physiol. 2003;284(1):C2-15.

31. Ballard ST, Spadafora D. Fluid secretion by submucosal glands of the tracheobronchial airways. Respir Physiol Neurobiol. 2007;159(3):271-277.

32. Salinas D, et al. Submucosal gland dysfunction as a primary defect in cystic fibrosis. FASEB J. 2005;19(3):431-433.

33. Engelhardt JF, et al. Submucosal glands are the predominant site of CFTR expression in the human bronchus. Nat Genet. 1992;2(3):240-248.

34. Liu L, et al. Method for quantitative study of airway functional microanatomy using micro-optical coherence tomography. PLoS ONE. 2013;8(1):e54473.

35. Trout L, King M, Feng W, Inglis SK, Ballard ST. Inhibition of airway liquid secretion and its effect on the physical properties of airway mucus. Am J Physiol. 1998;274(2 Pt 1):L258-L263.

36. Tang XX, et al. Acidic pH increases airway surface liquid viscosity in cystic fibrosis. J Clin Invest. 2016;126(3):879-891.

37. Martens CJ, Inglis SK, Valentine VG, Garrison J, Conner GE, Ballard ST. Mucous solids and liquid secretion by airways: studies with normal pig, cystic fibrosis human, and non-cystic fibrosis human bronchi. Am J Physiol Lung Cell Mol Physiol. 2011;301(2):L236-L246.

38. Matsui $\mathrm{H}$, et al. A physical linkage between cystic fibrosis airway surface dehydration and Pseudomonas aeruginosa biofilms. Proc Natl Acad Sci USA. 2006;103(48):18131-18136.

39. Henderson AG, et al. Cystic fibrosis airway secretions exhibit mucin hyperconcentration and increased osmotic pressure. JClin Invest. 2014;124(7):3047-3060.

40. Birket SE, et al. Combination therapy with cystic fibrosis transmembrane conductance regulator modulators augment the airway functional microanatomy. Am J Physiol Lung Cell Mol Physiol. 2016;310(10):L928-L939.

41. Chu KK, et al. Particle-tracking microrheology using micro-optical coherence tomography. Biophys J. 2016;111(5):1053-1063.

42. Cooper JL, Quinton PM, Ballard ST. Mucociliary transport in porcine trachea: differential effects of inhibiting chloride and bicarbonate secretion. Am J Physiol Lung Cell Mol Physiol. 2013;304(3):L184-L190.

43. Ballard ST, Trout L, Garrison J, Inglis SK. Ionic mechanism of forskolin-induced liquid secretion by porcine bronchi. Am J Physiol Lung Cell Mol Physiol. 2006;290(1):L97-104.

44. Pier GB. The challenges and promises of new therapies for cystic fibrosis. J Exp Med. 2012;209(7):1235-1239.

45. Reeves EP, Molloy K, Pohl K, McElvaney NG. Hypertonic saline in treatment of pulmonary disease in cystic fibrosis. Scien tificWorldJournal. 2012;2012:465230.

46. Rosenfeld M, et al. Inhaled hypertonic saline in infants and children younger than 6 years with cystic fibrosis: the ISIS randomized controlled trial. JAMA. 2012;307(21):2269-2277.

47. Subbarao $\mathrm{P}$, et al. Lung clearance index as an outcome measure for clinical trials in young children with cystic fibrosis. A pilot study using inhaled hypertonic saline. Am J Respir Crit Care Med. 2013;188(4):456-460.

48. Schultz BD. Airway epithelial cells: 'Bicarbonate in' $\neq$ 'Bicarbonate out'. J Physiol (Lond). 2012;590(21):5263-5264.

49. Leinonen JS, Saari KA, Seppänen JM, Myllylä HM, Rajaniemi HJ. Immunohistochemical demonstration of carbonic anhydrase isoenzyme VI (CA VI) expression in rat lower airways and lung. J Histochem Cytochem. 2004;52(8):1107-1112.

50. Song Y, Salinas D, Nielson DW, Verkman AS. Hyperacidity of secreted fluid from submucosal glands in early cystic fibrosis. Am 
J Physiol, Cell Physiol. 2006;290(3):C741-C749.

51. Zuelzer WW, NewTON WA. The pathogenesis of fibrocystic disease of the pancreas; a study of 36 cases with special reference to the pulmonary lesions. Pediatrics. 1949;4(1):53-69.

52. Stenbit AE, Flume PA. Pulmonary exacerbations in cystic fibrosis. Curr Opin Pulm Med. 2011;17(6):442-447.

53. Moskowitz SM, Gibson RL, Effmann EL. Cystic fibrosis lung disease: genetic influences, microbial interactions, and radiological assessment. Pediatr Radiol. 2005;35(8):739-757.

54. Harun SN, Wainwright C, Klein K, Hennig S. A systematic review of studies examining the rate of lung function decline in patients with cystic fibrosis. Paediatr Respir Rev. 2016;20:55-66.

55. Goss CH, Newsom SA, Schildcrout JS, Sheppard L, Kaufman JD. Effect of ambient air pollution on pulmonary exacerbations and lung function in cystic fibrosis. Am J Respir Crit Care Med. 2004;169(7):816-821.

56. Burgel PR, Montani D, Danel C, Dusser DJ, Nadel JA. A morphometric study of mucins and small airway plugging in cystic fibrosis. Thorax. 2007;62(2):153-161.

57. Ermund A, et al. The normal trachea is cleaned by MUC5B mucin bundles from the submucosal glands coated with the MUC5AC mucin. Biochem Biophys Res Commun. 2017;492(3):331-337.

58. Ostedgaard LS, et al. Gel-forming mucins form distinct morphologic structures in airways. Proc Natl Acad Sci USA 2017;114(26):6842-6847.

59. Stoltz DA, Meyerholz DK, Welsh MJ. Origins of cystic fibrosis lung disease. N Engl J Med. 2015;372(4):351-362.

60. VanDevanter DR, Kahle JS, O'Sullivan AK, Sikirica S, Hodgkins PS. Cystic fibrosis in young children: A review of disease manifestation, progression, and response to early treatment. J Cyst Fibros. 2016;15(2):147-157.

61. Donnelley M, Morgan KS, Awadalla M, Farrow NR, Hall C, Parsons DW. High-resolution mucociliary transport measurement in live excised large animal trachea using synchrotron X-ray imaging. Respir Res. 2017;18(1):95

62. Ermund A, Meiss LN, Gustafsson JK, Hansson GC. Hyper-osmolarity and calcium chelation: Effects on cystic fibrosis mucus Eur J Pharmacol. 2015;764:109-117.

63. Ermund A, et al. OligoG CF-5/20 normalizes cystic fibrosis mucus by chelating calcium. Clin Exp Pharmacol Physiol. 2017;44(6):639-647.

64. Abdullah LH, et al. Defective postsecretory maturation of MUC5B mucin in cystic fibrosis airways. JCI Insight. 2017;2(6):e89752.

65. Yuan S, et al. Oxidation increases mucin polymer cross-links to stiffen airway mucus gels. Sci Transl Med. 2015;7(276):276ra27.

66. Dawson M, Wirtz D, Hanes J. Enhanced viscoelasticity of human cystic fibrotic sputum correlates with increasing microheterogeneity in particle transport. J Biol Chem. 2003;278(50):50393-50401. 